Meta

Journal des traducteurs

Translators' Journal

\title{
Petit glossaire nordique du Canada
}

\section{Jean Hamelin}

Volume 19, numéro 3, septembre 1974

URI : https://id.erudit.org/iderudit/003001ar

DOI : https://doi.org/10.7202/003001ar

Aller au sommaire du numéro

Éditeur(s)

Les Presses de l'Université de Montréal

ISSN

0026-0452 (imprimé)

1492-1421 (numérique)

Découvrir la revue

Citer cet article

Hamelin, J. (1974). Petit glossaire nordique du Canada. Meta, 19(3), 154-158.

https://doi.org/10.7202/003001ar d'utilisation que vous pouvez consulter en ligne.

https://apropos.erudit.org/fr/usagers/politique-dutilisation/ 


\section{PETIT GLOSSAIRE NORDIQUE DU CANADA}

La majorité des termes sont des néologismes qui sont apparus au cours des quinze dernières années et qui peuvent témoigner de l'évolution dans la conceptualisation de la nordicité. Certains mots expriment une néologie totale, c'est-à-dire de forme et de sens (VAPO), d'autres, une création lexicale (GLACIEL) ou seulement une modification morphologique (KABLOUNISATION) et une addition sémantique (NORDIQUE). Le terme français est indiqué en vedette, suivi de son équivalent anglais.

ALBEDO ( $A L B E D O)$ : Réflectivité d'une surface par rapport à la venue d'ondes lumineuses ou calorifiques. L'albedo décourage l'absorption de la chaleur immigrante. Exemple, le tapis nival défavorise la pénétration de la chaleur solaire. S'exprime en pourcentage des pertes.

AMÉRINDIANISATION (AMERINDIANIZATION) : Processus historiques ou de reprise suivant lesquels s'accentuent dans un objet ou un sujet les caractères indiens ou inuit. L'inverse connaît une «désamérindianisation 》 ou érosion de ces traits. Mot et les suivants de même famille développés à partir de Amerind (J. W. Powell, 1899).

AMÉRINDIANITÉ ( $A M E R I N D I A N I T Y$ ) : Exprime l'état d'être amérindien. Concerne aussi bien la terre, les habitants, l'emploi que les faits culturels. Évolue dans le temps. Montre différents niveaux.

AMÉRINDIANYME ( $A M E R I N D I A N Y M$ ) : Nom de lieu issu de l'une ou l'autre des langues amérindiennes. Nahanni est un amérindianyme.

AMÉRINDIE (AMERINDIA) : Aires culturelles des Inuit et des autres ethnies indigènes dans les Amériques. La partie canadienne de l'Amérindie s'étend sur la presque totalité du Canada.

AMÉRINDIEN ( $A M E R I N D I A N)$ : De Amerind : Indigène des Amériques. Esquimau (Inuk) et Indien. Considéré surtout aux plans aréal, ethnique et linguistique.

AMÉRINDIOLOGIE ( $A M E R I N D I O L O G Y$ ) : Étude complète et interdisciplinaire de tous les aspects concernant les Indiens et les Inuit.

AMÉRINDOÏDE ( $A M E R I N D O I D)$ : Qui suggère des formes et un faciès indien ou inuk.

AMÉRINDOPHONE (Amerindophone) : Souligne l'identité des langues indiennes et esquimaude. S'applique d'abord aux individus et, par extension, aux aires et faits culturels. Aklavik est un nom de lieu amérindophone.

ARÉAL ( $A R E A L)$ : Se dit d'une surface. Évite la confusion avec territorial, terme politique du Nord canadien.

CANADA DE BASE ( $B A S E C A N A D A$ ) : La région ainsi désignée occupe la partie méridionale du Canada, du sud de la Colombie britannique à l'île de 
Terre-Neuve. Vue zonalement, elle comprend les foyers principaux de l'écoumène canadien ainsi que la zone contiguë du pré Nord. Ce qui n'est pas «Canada de base » compose le Nord proprement dit. Les régions maîtresses du Canada de base constituent autant de points d'appui dans le développement du Nord.

CANADIANITÉ (CANADIANITY) : Exprime les caractères d'identité du Canada. Suggère un état d'âme, une mentalité, une maturation. Pour une part, la jeunesse du pays rend compte des limites de la canadianité. Différent de canadianisation qui s'applique aux processus.

CHORONYMIE (CHORONYMY) : Totalité des études qui concernent l'appellation passée et future de toute surface aréale. Englobe plus que la toponymie traditionnelle.

DIEL $(D I A L)$ : Se dit d'une situation de 24 heures. Dans le haut Arctique où le soleil ne se lève pas durant l'hiver, l'obscurité dielle constitue l'un des caractères les plus pénibles; au contraire, durant l'été, le soleil ne se couche pas et c'est l'éclairement qui est diel. Diel n'équivaut pas à diurne, ce dernier étant opposé à nocturne.

ÉCOUMÈNE ou OÉCOUMÈNE (ECUMENE) : Territoire en fonction de l'emprise des hommes. Les principales fonctions de l'écoumène concernent la résidence, l'exploitation et les liaisons. Les zones les moins écouménées sont des môles répulsifs ou non attractifs. Sur le plan des rendements, l'écoumène peut être dit extensif ou intensif; dans le Nord, il est surtout extensif.

ENGEL (FREEZE-UP; FREEZING) : Dans le cycle du gel, moment de l'installation de l'état de gel dans un matériau; parallèle à regel mais pour un cycle antérieur. Phase opposée à celle du dégel.

EXTRÊME-NORD (EXTREME NORTH) : Niveau hyperpolaréen d'une région. Zone de nordicité maximale (au-delà de 800 VAPO). Partie septentrionale de l'archipel arctique. Généralement présence de glaciers, glaces flottantes et glace de sol. Au Canada, zone artificiellement peuplée de quelques centaines d'habitants seulement.

GLACIEL ( $G L A C I E L$ ) : Qualifie tout ce qui se rapporte aux glaces flottantes, p.e. le pack est une couverture glacielle. Dans les pays froids, la période sans glace sur eau pourrait être désignée interglacielle.

GRAND NORD ( FAR NORTH) : Niveau mégapolaréen d'une région. Zone de nordicité élevée (de 500 à 800 VAPO) mais non maximale. Au Canada, elle s'étend surtout dans les Territoires du Nord-Ouest et correspond en partie au climat arctique. 20000 habitants seulement dont une majorité d'Inuit. Frobisher Bay et Inuvik constituent les principales agglomérations qui sont localisées dans cette zone.

HUDSONIE (HUDSONIA) : Régionyme (nom de région) établi à partir du mot Hudson. Deux types d'Hudsonie :

1) L'Hudsonie maritime comprend la mer d'Hudson, la baie de James, le détroit d'Hudson, la baie d'Ungava et le golfe de Foxe. 
2) L'Hudsonie commerciale englobe en outre tout le bassin hydrographique qui composait le principal territoire de cueillette des fourrures de la Compagnie de la Baie d'Hudson.

INDICE NORDIQUE (INDEX OF NORTHERNNESS) : Outil de calcul permettant de quantifier le nombre de valeurs polaires (VAPO) et partant d'établir la nordicité d'un espace. Cet indice est basé sur 10 critères et s'exprime en unités par rapport à un maximum de 1000 .

INUK (INUK) : Nom que l'Esquimau se donne. Par extension, l'Homme. Pluriel : Inuit. Formation possible de mots dérivés.

INUKLAND (INUKLAND) : Domaine parcouru par les Inuit; il s'étend surtout dans la zone du Grand Nord. Dans le passé, l'extension de l'Inukland a varié.

ISONORD (ISONORD) : Sur une carte, trait réunissant les points d'égale nordicité. Même méthode que dans le cas des pressions, des pluies et des températures. L'on peut dire isovapo (voir VAPO).

KABLOUNISATION (KABLOONIZATION) : De Kabloona (i1 y a 10 façons d'écrire le mot) : homme ayant de grands sourcils ou homme blanc ou tout autre qu'un Inuk. Chez certains Inuit, effort personnel de mutation dans le but d'afficher des comportements de Blanc ou en réponse à des sollicitations peu motivées. (Développé à partir de Kabloonamiut, F. A. Vallée, 1962.)

MOYEN NORD (MIDDLE NORTH) : Niveau mésopolaréen d'une région. Zone de moyenne nordicité (de 200 à 500 VAPO) s'étirant du Labrador au Yukon. Elle s'étend en partie sur 7 provinces et 2 territoires. Cette zone de présence pionnière rassemble environ un quart de million d'habitants. D'importantes villes dont Thompson, Whitehorse et Yellowknife. Le Moyen Nord, large d'environ 500 milles $(800 \mathrm{~km})$ peut se diviser en deux : au sud, un Bas Moyen Nord, au nord, un Haut Moyen Nord.

NORD CANADIEN (CANADIAN NORTH) : Comprend la partie septentrionale de sept provinces, le Yukon et les territoires du Nord ainsi que des nappes d'eau marines « intérieures» comme la mer d'Hudson. Est limité au sud par l'isoligne de 200 VAPO. Voir carte. Zonalement, se divise en Moyen Nord, Grand Nord et Extrême Nord.

NORD CORDILLÉRAN (CORDILLERAN NORTH) : Vu longitudinalement, le Nord canadien peut être partagé en fuseaux orientés nord-sud. La partie du Nord située à l'intérieur des Cordillères compose le Nord cordilléran qui s'étend surtout en Colombie et au Yukon. Outre les montagnes et vallées, la route Alcan structure un secteur de cette région.

NORDICITÉ (NORDICITY) : Nom donné à la «polaricité » de l'hémisphère boréal. État et quantité nordique (nombre de VAPO) d'un lieu, d'un caractère et même d'une population. Différents degrés dans la nordicité à partir d'un point 0 , celui d'« anordicité $»$. 
NORDISME (NORDISM) : Ensemble des attitudes mentales ou des activités exprimant un engagement en faveur du Nord.

NORDIQUE (NORDIC) : Néologisme de sens qui peut s'appliquer non seulement aux régions, caractères et habitants du Nord de l'Europe mais à celles et ceux situés dans toute la partie septentrionale de l'hémisphère boréal. Universalisation du sens d'une expression connue. Par provignement, «nordiquement», « intranordique....»

NORDISTE (NORDIST - NORTHERNER) : Personne qui porte référence au Nord; il réside généralement dans le Nord. Les Nordistes comprennent à la fois des Indigènes et des non-Indigènes. Certains sont des "Néo-Nordistes 》. Un Nordiste par localisation n'équivaut pas nécessairement à un Nordiste de mentalité.

NUNARITÉ (NUNARITY) : De Nuna : la terre; son pays. a) État de vie des Inuit qui est conforme aux valeurs traditionnelles; menacé par la « kablounisation ». b) Nunamiut, le peuple de la terre ou les Inuit du caribou.

PACK $(P A C K)$ : Banquise de glaces flottantes, généralement mouvantes et occupant toute l'année la grande partie de l'océan Arctique. Par extension, champs saisonniers de glaces flottantes; par exemple, dans la mer d'Hudson. Cette couverture de glace est souvent conglomératique et à surface non uniforme. Le pack est l'une des caractéristiques du glaciel.

PÉTROLERIE (OIL INDUSTRY) : Néologisme de sens. Ensemble des activités et des paysages d'extraction, de fractionnement, de distillation, de transport, d'entreposage et de consommation du pétrole. Le tout s'accompagnant de formes de pollution. Concerne également d'autres aspects comme le financement, l'emploi, les profits et ce qui reste à la fin des opérations.

PRÉ NORD (NEAR NORTH) : Niveau sous-polaréen d'une région froide. Zone faiblement nordique, sise au sud du Nord proprement dit et en liaison avec les foyers plus animés du Sud canadien. Terre-Neuve (en bonne partie), l'Abitibi, le lac Supérieur et la Rivière-de-la-Paix sont dans le pré Nord. Au Canada, la zone comprend quelques millions d'habitants.

RÉGIOLOGIE (REGIOLOGY) : L'étude des régions à tous les points de vue. Se distingue de la régionalisation qui est le fait propre de reconnaître des aires et, par extension, le résultat de cette action. La « régionymie», partie de la choronymie, étudie le nom des régions, grandes ou petites. Dans le Nord, les recherches régiologiques ont fait voir des zones, telle celle du «Moyen Nord》.

S'ANORDIR (NORTHING) : Vieux mot signifiant l'accroissement des caractères nordiques (ou de la nordicité) chez un individu, dans une mentalité, dans une structure, à l'intérieur d'une région.

SUDISTE (SOUTHERNER) : Au Canada, personne qui sur le plan des mentalités ou des activités ne porte pas référence au Nord du pays. 
TERRITORIAL (TERRITORIAL) : Qualifiant la situation politique (antéprovinciale ou quasi-provinciale ou mi-provinciale) de l'un et de l'autre des deux Territoires nordiques du Canada. Par dérivation : «territorialisation».

TERRITORIEN (TERRITORIAN) : Qualifie une situation en rapport avec les Territoires du Nord-Ouest seulement; parallèlement à Yukonien en ce qui concerne le Territoire de Yukon. Extension moins grande que celle de territorial.

VAPO (VAPO) : Sigle pour «valeurs polaires». Unité qui permet d'exprimer la nordicité. Le pôle a 1000 VAPO ; Churchill au Manitoba, près de 500. Ces VAPO sont calculées au moyen d'un indice polaire (ou indice nordique dans l'hémisphère boréal).

JEAN HAMELIN 


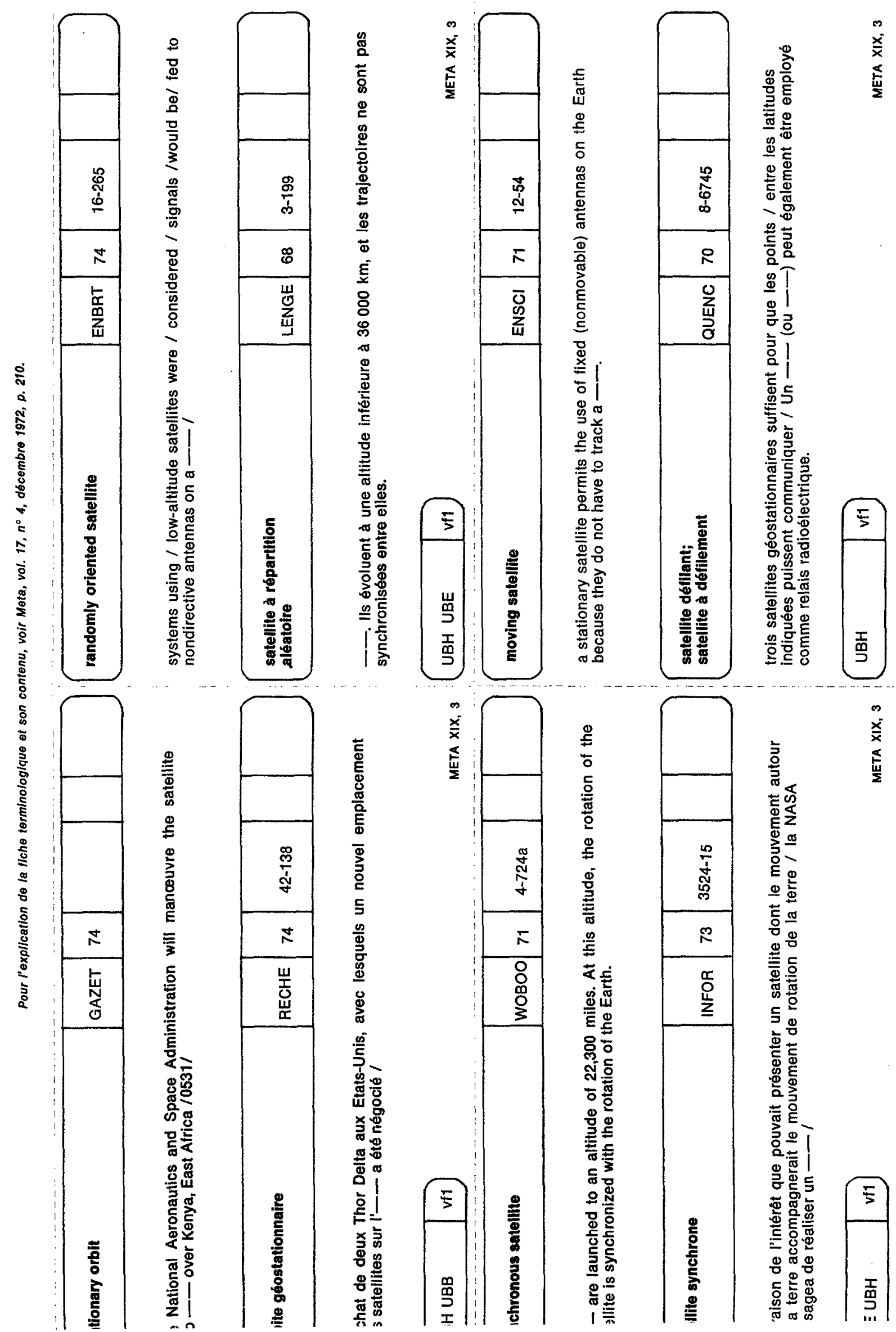

